\title{
ANÁLISIS DE FLUJOS DE AGUA EN ÁREAS METROPOLITANAS DESDE LA PERSPECTIVA DEL METABOLISMO URBANO
}

\author{
María Isabel García Serna ${ }^{1}$ \\ Tito Morales-Pinzón ${ }^{2}$ \\ Jhoniers Guerrero Erazo ${ }^{3}$
}

Recibido el 30 de agosto de 2013 y aprobado el 15 de enero de 2014, actualizado el 11 junio de 2014

\begin{abstract}
RESUMEN
Las áreas urbanas demandan flujos intensos de agua para su funcionamiento. EI análisis de su estado es mejor entendido a partir de estudios metabólicos que permiten analizar los flujos de agua que entran y salen del sistema urbano. Objetivo: Cuantificar los flujos de agua del Área Metropolitana Centro Occidente (Colombia), determinando la magnitud de sus demandas y el grado de exigencia de la ciudad sobre el recurso agua. Método: Se utilizó como herramienta metodológica el análisis de flujos de materiales para cuantificar los flujos de entrada, intermedios y de salida del sistema urbano. Resultados: En el área de estudio, el $86 \%$ del agua es abastecida por el río Otún como principal fuente local y el $14 \%$ restante es abastecido de fuentes hídricas externas. El agua derivada anualmente para el abastecimiento urbano de sus 599.618 habitantes alcanzó los 31,2 MMC/año, es decir 113 I/hab/día. Con relación a los flujos de salida se destaca cómo las aguas residuales se vierten directamente a las fuentes hídricas sin ningún tipo de tratamiento previo. El balance de materia determinó un flujo volumétrico de agua para el año 2011 de 155,2 MMC/año que entran y salen de la región metropolitana. Conclusión: En el Área Metropolitana Centro Occidente la gestión del recurso hídrico obedece a un proceso lineal (captación, distribución, consumo y vertimientos) generando desechos no tratados que son reintroducidos al ciclo ecosistémico. La demanda y el consumo de agua tienen una tendencia decreciente en oposición al crecimiento poblacional, indicando un mejor uso racional del recurso o una estrategia de adaptación al costo del servicio público.
\end{abstract}

\section{PALABRAS CLAVE}

Áreas metropolitanas, flujos de agua, metabolismo urbano.

\section{ANALYSIS OF WATER FLOWS IN METROPOLITAN AREAS FROM THE URBAN METABOLISM PERSPECTIVE}

\begin{abstract}
Urban areas require intense water flows in order to operate. The analysis of the state of such water flows is better understood from metabolic studies that allow analyzing water flows entering and leaving the urban system. Objective: Quantify water flows of the Colombian Central-Western Metropolitan Área, determining the extent of its demands and the strictness of the city on water resources. Method: The material flow analysis was used as methodological tool to quantify the urban system inflows, intermediate flows and output flows. Results: In the area studied, $86 \%$ of the water is supplied by the Otún river as the main local source and the remaining $14 \%$ is supplied by external water sources. The water diverted annually for the urban supply of its 599.618 inhabitants consumption reached 31.2 MMC/year that is $113 \mathrm{l} /$ person/day. Regarding outflows it is important to highlight
\end{abstract}


that wastewater is discharged directly into water sources without any pretreatment. The material balance determined a volumetric water flow for 2011 of 155.2 MCM/year that enters and leaves the metropolitan region. Conclusion: In the Central-Western Metropolitan Área water resource management follows a linear process (collection, distribution, consumption and discharges) generating untreated waste streams that are reintroduced into the ecosystem cycle. The demand and consumption of water have a decreasing trend in opposition to population growth, indicating better rational use of resources or an adaptation strategy to the public service cost.

\section{KEY WORDS}

Metropolitan areas, urban metabolism, water flows.

\section{INTRODUCCIÓN}

El mundo se urbaniza cada vez más; cuatro de cada cinco personas en la región de América Latina y el Caribe viven en ciudades (ONU-HÁBITAT, 2009). El rápido y desorganizado proceso de urbanización tiene impactos sustanciales en la disponibilidad y la calidad del agua dentro de los límites internos y externos de las ciudades, entre los cuales se incluyen la sobreexplotación de los recursos hídricos (superficiales y subterráneos), la menor seguridad del agua, la mayor vulnerabilidad de que se produzcan inundaciones e impactos en la salud relacionados con el agua (Banco Mundial, 2012).

Algunas ciudades de América Latina y el Caribe dependen de una única fuente de abastecimiento y en otros casos es necesario trasladar volúmenes importantes de agua desde zonas más lejanas, con mayores costos económicos y ambientales debido a que las fuentes hídricas son escasas o no garantizan cantidad y calidad, tal es el caso de ciudades como Ciudad de México en Centroamérica, el litoral de Perú, el nordeste de Brasil, el altiplano de Bolivia, el sector de Cuyo y el sur de Argentina, entre otras regiones. El problema es especialmente agudo en las grandes áreas urbanas, como en la Ciudad de México y Lima (ONU-HÁBITAT, 2012).

A la explotación excesiva del agua disponible, se suma la contaminación del recurso por vertido de aguas residuales, que reciben insuficiente tratamiento; se estima que en los países de América del Sur y el Caribe, menos del $20 \%$ de las aguas residuales son tratadas antes de su vertido (PNUMA, 2000). Muchos de los ríos urbanos presentan un alto grado de contaminación y deterioro natural, tal es el caso de la cuenca Matanza Riachuelo en Buenos Aires (Argentina) o el río Bogotá (Colombia).

En consecuencia, los territorios altamente urbanizados soportan flujos intensos cuya dirección es horizontal, extrayendo recursos del sistema natural que luego son transformados y consumidos, generando permanentemente corrientes de desecho que en ningún momento se gestionan o procesan para ser recirculadas al ciclo ecosistémico, todo lo contrario, estos son dispuestos de forma inadecuada.

Para analizar los flujos de agua que entran y salen del sistema urbano se requiere de la comprensión del concepto metabolismo que implica la reflexión sobre la ciudad y su funcionamiento como un organismo vivo, compuesto por 
células urbanas cuyas reacciones y procesos se basan en el intercambio de flujos de materia y energía que entran, salen y se transforman (Calderón, 2011).

Autores como Niza, Rosado y Ferrao (2009, p. 387) afirman que el metabolismo urbano puede "[...] ser usado como herramienta en la identificación de problemas ambientales relacionados con el crecimiento de las entradas, la gestión de las salidas y el diseño más eficiente de las políticas de planeamiento urbano", lográndose además determinar las características de disponibilidad natural de los recursos (Girardet, 2004), obtener una mayor comprensión de la forma en la que evolucionan las demandas de recursos de una ciudad (Stimson, Westem, Mullins y Simpson, 1999) y el deterioro causado por su aprovechamiento (Brunner, 2007).

Para comprender el tema a tratar, se resalta el conocimiento aportado por estudios de metabolismo urbano enfocados especialmente a temas urbanos del agua. Autores como Hermanowicz y Asano (1999), Thériault y Laroche (2001), Gandy (2004), Hoekstra y Chapagain (2008), March y Saurí (2010) han resaltado la importancia del agua como elemento fundamental para la sostenibilidad de las áreas urbanas.

En Latinoamérica encontramos pocos estudios que aborden el tema de metabolismo urbano, con un enfoque especializado en cuantificación de flujos en los sistemas urbanos. Navarro (2010) trabajó sobre la problemática del agua urbana en la ciudad de Tijuana, cuantificando los flujos de agua en las etapas de abasto, distribución y consumo. Por su parte, Díaz (2011) desarrolló un estudio que permitió conocer el profundo cambio e incremento de los flujos de agua, alimentos, combustibles y energía de la ciudad de Bogotá (Colombia), durante seis momentos históricos de la capital colombiana. Otro, es el estudio realizado por Delgado, Campos y Rentería (2012) quienes hacen una presentación general sobre el estado de situación de las ciudades latinoamericanas y con una introducción a las evaluaciones metabólicas para ofrecer una primera aproximación integral comparativa de los flujos de entrada y salida de las megaurbes latinoamericanas (Ciudad de México, Sao Paulo, Río de Janeiro y Buenos Aires), este trabajo resalta cómo este tipo de evaluaciones en América Latina están prácticamente ausentes.

En este contexto, este articulo pretende analizar los flujos de agua que entran y salen de una región urbana; pues el agua puede ser considerado el recurso natural que más limita el crecimiento y desarrollo futuro de los centros urbanos especialmente de países no desarrollados (Morales, Flórez y Ramírez, 2011).

\section{MATERIALES Y MÉTODOS}

\section{Área de estudio}

El área de estudio seleccionada es el Área Metropolitana Centro Occidente (AMCO), localizada en la región centro-occidental de Colombia. El AMCO está conformado por el municipio de Pereira como el núcleo principal, con 459.667 habitantes en 2011. A su vez este núcleo municipal cuenta con dos municipios satélites los cuales constituyen la totalidad del AMCO. La población de Dosquebradas es de 191.070 habitantes y la de La Virginia es de 31.729 habitantes en 2011 (DANE, 2005). La Figura 1 muestra la ubicación del AMCO en el departamento de Risaralda (Colombia). 


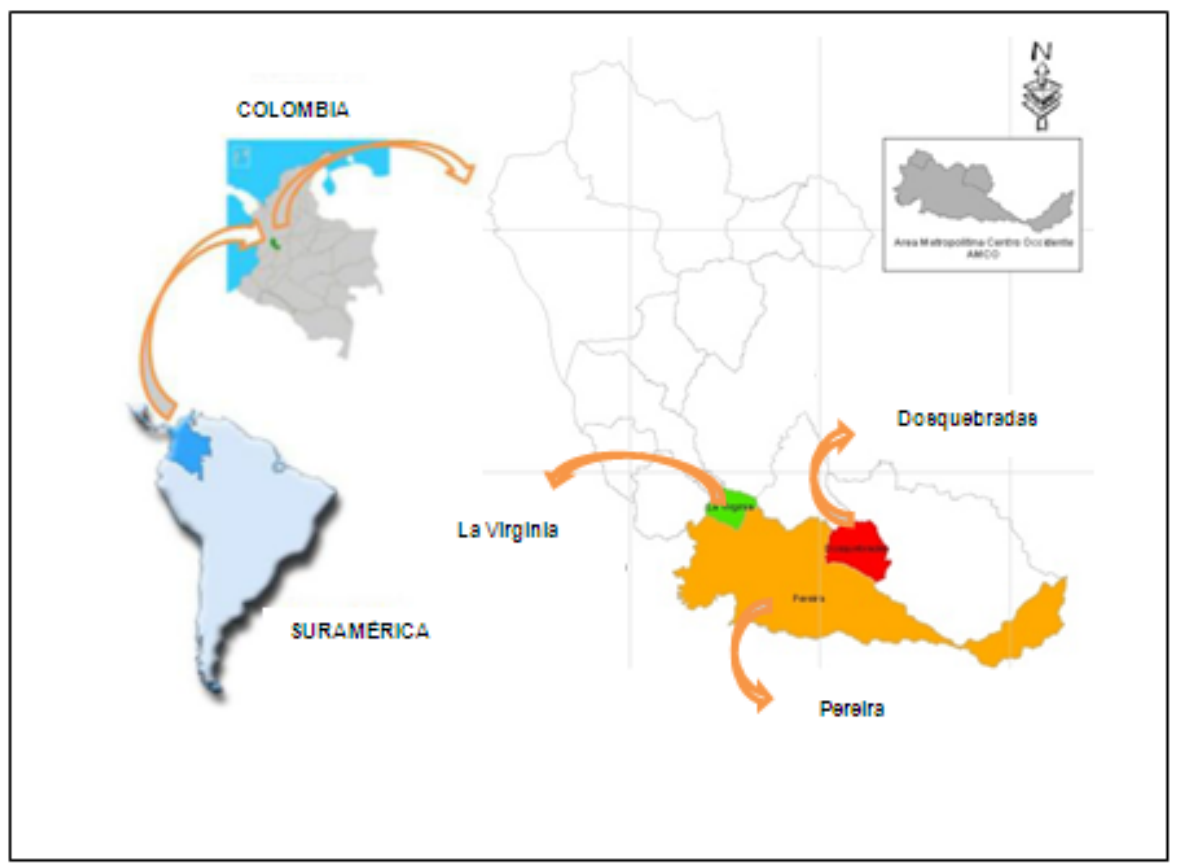

Fuente: Elaboración propia.

Figura 1. Localización área de estudio.

\section{Análisis de datos}

Se construyó una base de datos con información obtenida de los informes de gestión de las empresas de servicios públicos (Aguas y Aguas de Pereira, Serviciudad y Empresa de Servicios Públicos de La Virginia), el Sistema Único de Información (SUI) y la Superintendencia de Servicios Públicos Domiciliarios. EI espacio temporal escogido para el desarrollo del estudio fueron los años 2009, 2010 y 2011.

Se utilizó como herramienta metodológica el Análisis de Flujo de Materiales (AFM) que consiste en analizar los flujos de entrada que corresponden a precipitación, caudal captado de fuentes locales y externas. Los flujos intermedios contemplan producción, suministro y consumo de agua de los diferentes usos en el sistema urbano (residencial, industrial, comercial y oficial). Por último, los flujos de salida se concentran en el vertido de los diferentes usos en el sistema urbano (residencial, industrial, comercial y oficial) más escorrentía, pérdidas facturadas y no facturadas, infiltración y la evapotranspiración.

El cálculo del flujo de agua del AMCO se cuantifica a través de balances de materia que se representa mediante la siguiente ecuación: 


\section{Ecuación 1. Balance hídrico para el AMCO}

$\Sigma(P+F l+F e+F s)=\Sigma(D r+D i+D c+D o)=\Sigma\left(V r+V i+V_{c}+V_{0}+P_{e r}+E s u p+I+E\right)$

Donde:

\begin{tabular}{|c|c|}
\hline$P$ : & Precipitación (m³/año). \\
\hline $\mathrm{Fl}$ : & Fuentes locales ( $\mathrm{m}^{3} / \mathrm{año}$ ). \\
\hline $\mathrm{Fe}$ : & Fuentes externas ( $\left.\mathrm{m}^{3} / a n ̃ o\right)$. \\
\hline Fs: & Fuentes subterráneas ( $\left.\mathrm{m}^{3} / a n ̃ o\right)$. \\
\hline Dr: & Demanda residencial (m³/año). \\
\hline Di: & Demanda industrial ( $\left.\mathrm{m}^{3} / a n ̃ o\right)$. \\
\hline Dc: & Demanda comercial (m³/año). \\
\hline Do: & Demanda oficial (m³/año). \\
\hline $\mathrm{Vr}$ : & Vertido residencial ( $\mathrm{m}^{3} / \mathrm{año}$ ). \\
\hline Vi: & Vertido industrial ( $\mathrm{m}^{3} / \mathrm{año}$ ). \\
\hline Vc: & Vertido comercial (m³/año). \\
\hline Vo: & Vertido oficial (m³/año). \\
\hline Per & Pérdidas reales y aparentes ( $\mathrm{m}^{3} / \mathrm{año}$ ) \\
\hline Esup: & Escorrentía superficial (m³/año). \\
\hline & Infiltración (m³/año). \\
\hline$E$ : & evapotranspiración (m³/año). \\
\hline
\end{tabular}

\section{RESULTADOS Y DISCUSIÓN}

\section{Fuentes de agua para consumo humano}

La red de suministro de agua potable en el AMCO es manejada por diferentes empresas de servicios públicos, y cada uno de los municipios que pertenecen al centro metropolitano se abastece de diferentes fuentes superficiales. La principal fuente de abastecimiento de agua superficial del área urbana del municipio de Pereira es el río Otún, con un caudal promedio de $12,19 \mathrm{~m}^{3} / \mathrm{s}$, de los cuales se captan $2 \mathrm{~m}^{3} / \mathrm{s}$ con destino al acueducto de Pereira (Cárdenas, 2013). Esta cuenca atiende las demandas presentes y se visiona en la planificación de largo plazo, como la única alternativa segura de agua para la ciudad de Pereira.

Por otra parte, el área urbana del municipio de Dosquebradas no se abastece de fuentes superficiales propias de su territorio ya que no garantizan cantidad y calidad, por lo tanto importan agua de territorios vecinos como lo son el río Campoalegre del municipio de Santa Rosa de Cabal y el río Otún del municipio de Pereira. Con la compra de agua en bloque Dosquebradas abastece el $70 \%$ de su población y el otro $30 \%$ de sus habitantes solucionan su abastecimiento a través de acueductos de tipo comunitarios y privados (Quintana, 2009).

El municipio de La Virginia importa agua del río Totuí, subcuenca que hace parte de los municipios vecinos de Balboa, La Celia y Santuario. El caudal promedio de la cuenca es de $1,5 \mathrm{~m}^{3} / \mathrm{s}$, de los cuales el acueducto del municipio de La Virginia capta $0,18 \mathrm{~m}^{3} / \mathrm{s}$ para suplir los requerimientos de agua de la población.

\section{A) Flujos de entrada}

El AMCO depende el $86 \%$ del río Otún como su principal fuente local y $14 \%$ de fuentes hídricas externas que se traduce en importar agua para suplir sus necesidades (Figura 2). Existe una alta dependencia al río Otún como única fuente local abastecedora del área urbana de Pereira y parte del area urbana de 
Dosquebradas, generando presión sobre esta fuente hídrica ya que se capta un caudal promedio de 47,9 MMC/año para abastecer 153.825 usuarios. Los municipios de Dosquebradas y La Virginia garantizan disponibilidad y suministro de agua importando agua de cuencas vecinas, lo que les genera una condición de dependencia hídrica.

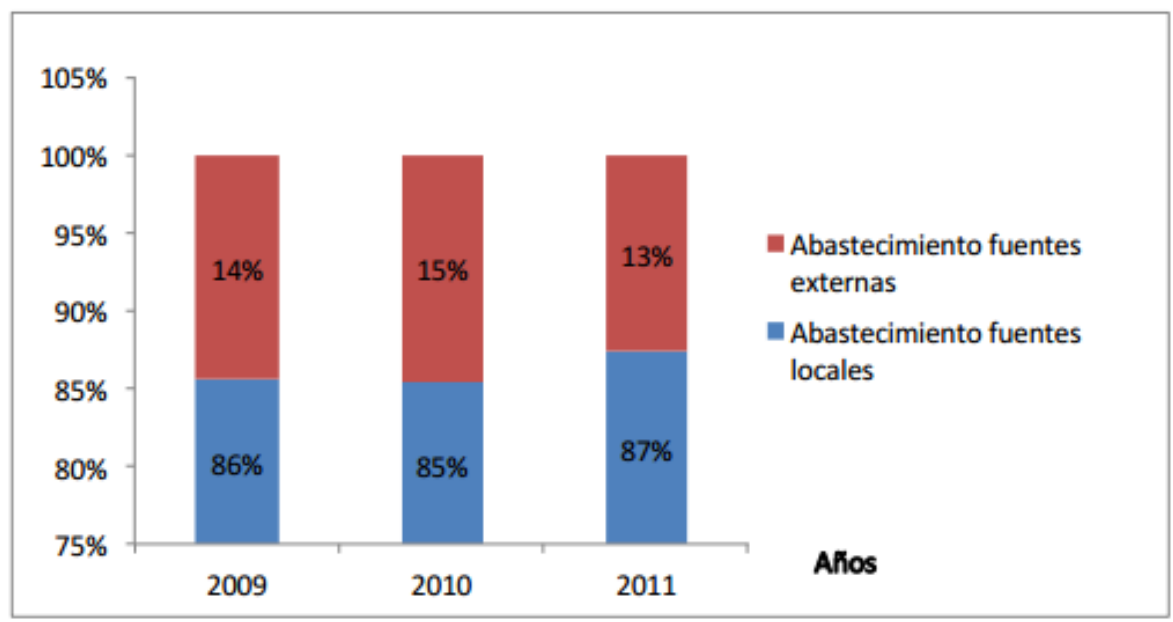

Fuente: Elaboración propia.

Figura 2. Origen fuentes hídricas que abastecen el AMCO.

La precipitación media anual del AMCO aportó un promedio de $656 \mathrm{~mm}$ para el año 2011. Descontando la evapotranspiración, se consiguió estimar la oferta pluvial urbana del AMCO (Figura 3).

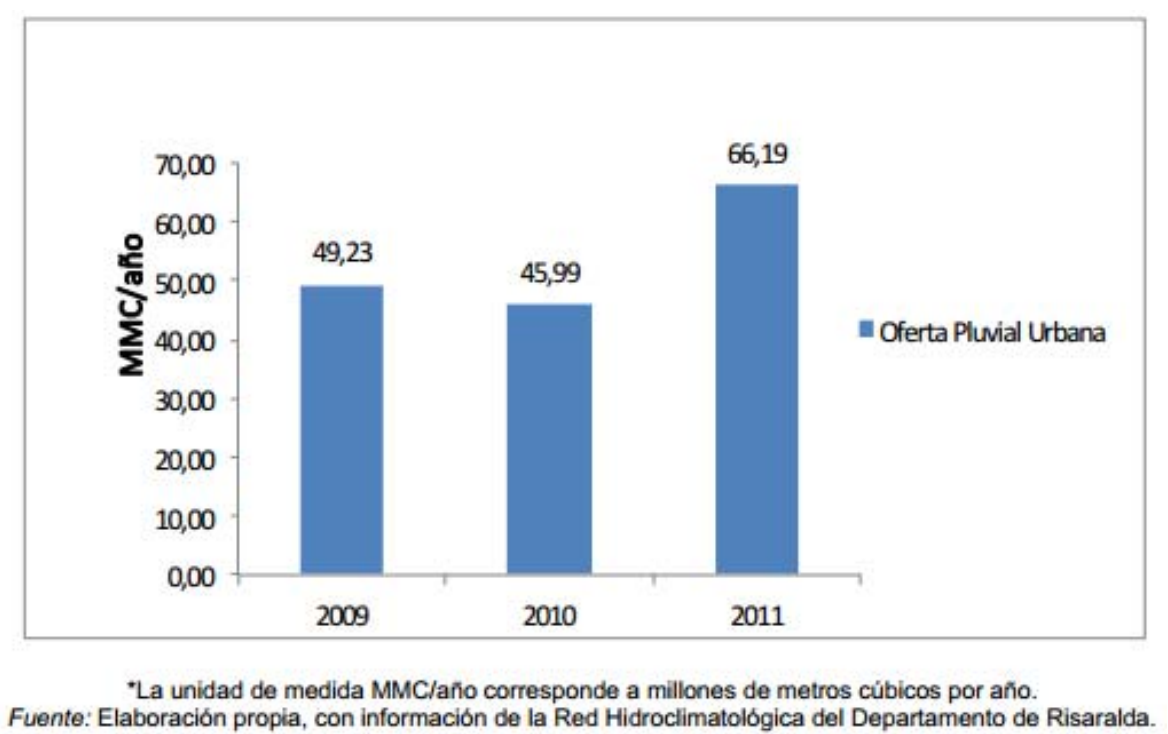

Figura 3. Oferta pluvial urbana del AMCO.

A pesar de la existencia de este gran potencial, en el AMCO, son pocos los casos documentados de aprovechamiento de agua pluvial, y es conocido que algunas empresas del sector industrial como las tintorerías, empresas que preservan madera y algunos edificios como el de la empresa telefónica de Pereira y centros comerciales hacen uso del agua lluvia para sus procesos. Sin 
embargo, en la región metropolitana la mayor parte del agua pluvial no se aprovecha y frecuentemente se contamina, pues al canalizarla y posteriormente conducirla al drenaje, el agua pluvial se mezcla con aguas negras, jabonosas y residuos industriales, convirtiéndose en agua contaminada.

En la Figura 4 se muestra la oferta de agua lluvia que, al ser comparada con el consumo total facturado, se muestra como una fuente susceptible a captación y puede ser una fuente alternativa de suministro de agua para el AMCO.

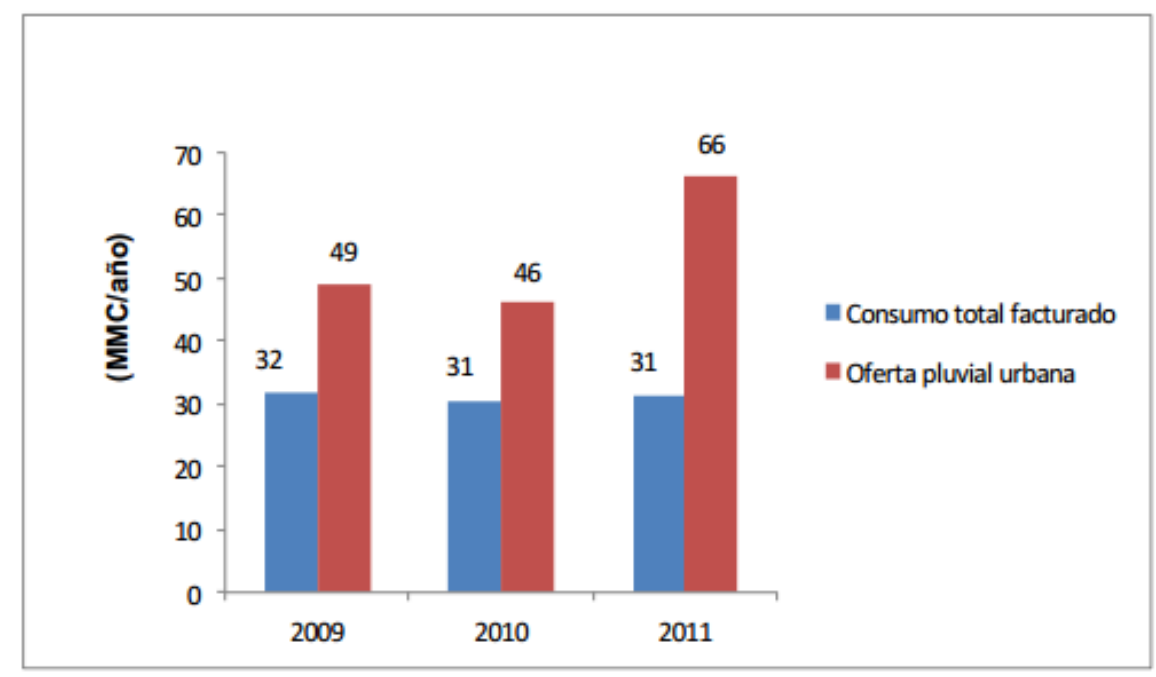

Fuente: Elaboración propia, con información del SUl e informes de gestión Empresas de Servicios Públicos.

Figura 4. Oferta pluvial urbana vs Consumo total facturado.

\section{B) Flujos intermedios}

Las cantidades de agua que intervienen en los abastecimientos de agua potable de una ciudad pueden clasificarse en tres grupos: 1) el agua captada, es decir la que se deriva de las fuentes aprovechadas para el servicio; 2) el agua suministrada, la parte del volumen captado que entra al sistema de distribución de la ciudad; y 3 ) el agua consumida, que es la recibida por el usuario para satisfacer sus necesidades (Gómez, 1980).

Los sistemas de abastecimiento del AMCO captaron en el año 2011 un promedio de 54,9 MMC/año (Figura 5), mientras que para el mismo año, el agua suministrada fue de 50,8 MMC/año, y el consumo facturado que es la cantidad que realmente suple las necesidades del sistema urbano alcanzó 31,2 MMC/año (Figura 5). Las variaciones se deben a dos causas principales: a las pérdidas aparentes que están conformadas por los consumos no autorizados y las imprecisiones de medición, mientras que las pérdidas reales son conformadas por las fugas ya sea en conducción o distribución, por derrames de tanques, y en tomas o conexiones de servicio antes del punto de medición de la conexión domiciliaria (Morales et al., 2011).

Las pérdidas aparentes y reales para el año 2011 fueron del orden de 25,0 MMC/año. La Figura 5 permite observar una disminución en el agua captada y facturada; y como consecuencia una disminución de las pérdidas del sistema pasando de 27,6 MMC/año en el 2009 a 25,0 MMC/año en el 2011. 


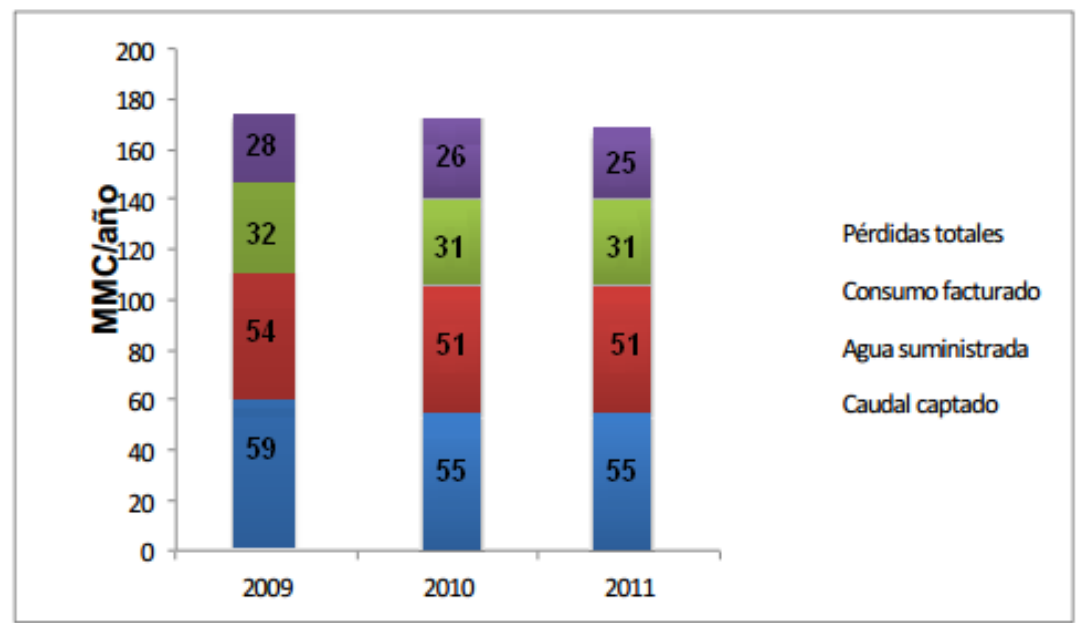

Fuente: Elaboración propia con información del SUI e informes de gestión Empresas de Servicios Públicos.

Figura 5. Captación, suministro, facturación y pérdidas de los sistemas de abastecimiento de agua en el AMCO.

La mayor demanda del recurso hídrico para el año 2011 se concentró en el sector residencial debido al crecimiento urbano, con un consumo promedio de 24,07 MMC/año, seguido por el comercial con 3,00 MMC/año, el oficial con 1,45 MMC/año y el industrial con 0,68 MMC/año (Figura 6). Los consumos de agua en el sector residencial, comercial y oficial muestran una disminución año a año.

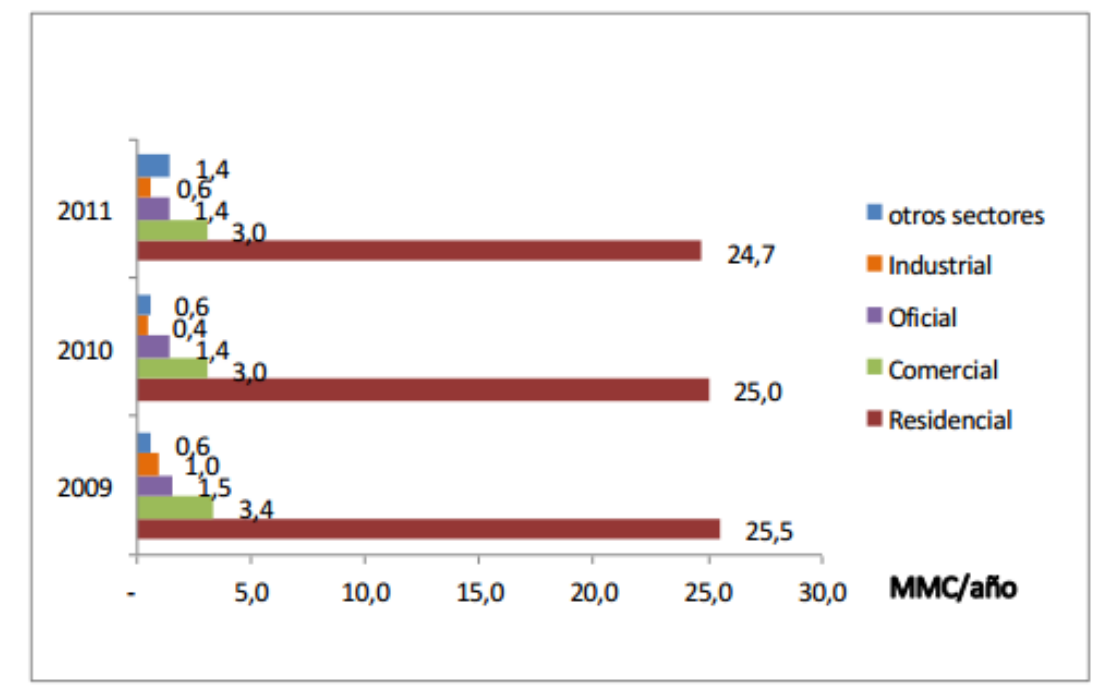

Fuente: Elaboración propia con información del SUl e informes de gestión Empresas de Servicios Públicos.

Figura 6. Evolución del consumo de agua facturada en el AMCO discriminada por sectores.

La dotación de agua (litros/habitante/día) en el AMCO, expresada en función del agua consumida en el sector residencial, se ha visto reducida en el periodo 20092011, pasando de 118 a 113 I/hab/día (Tabla 1). 
Tabla 1. Dotación de agua en el AMCO

\begin{tabular}{c|cc}
\hline Año & Población Hab & Dotación l/hab/dia \\
\hline 2009 & 590.370 & 118 \\
2010 & 594.997 & 115 \\
2011 & 599.618 & 113 \\
\hline
\end{tabular}

Fuente: Elaboración propia.

Si bien los factores que han contribuido al descenso del consumo de agua no están claramente identificados, se pueden considerar varios factores. Campbell, Larson, Johnson y Waits (1999) y Arbués, Barberán y Villanúa (2008), consideran dentro de sus hallazgos que el precio del agua puede ser el método de conservación más efectivo, es decir los aumentos de los precios de agua pueden determinar una baja en los consumos. Por su parte, Arbués et al. (2008) exponen que el tamaño de los hogares y el consumo total tienen relación directa, pues el consumo aumenta a medida que se incrementa el número de habitantes por vivienda. Los programas para uso eficiente y ahorro de agua también pueden producir cambios en los patrones o hábitos de consumo de los usuarios. En la Tabla 2 se presentan los valores facturados por metro cúbico para los diferentes sectores, mostrando cómo los precios del agua han aumentado en los últimos años, lo que indica que el aumento de los precios del agua inciden en la baja en los consumos aceptando las reflexiones de Campbell et al. (1999) y Arbués et al. (2008).

Tabla 2. Valor facturado por unidad de consumo

\begin{tabular}{c|ccc}
\hline \multicolumn{5}{c}{ Valor facturado por unidad de consumo $\left(\mathbf{s} / \mathrm{m}^{\mathbf{}}\right)$} & 2011 \\
\hline Estrato & 2009 & 2010 & 1.118 \\
\hline Estrato 1 & 1.050 & 1.082 & 1.112 \\
Estrato 2 & 1.042 & 1.078 & 1.115 \\
Estrato 3 & 1.039 & 1.076 & 1.110 \\
Estrato 4 & 1.028 & 1.063 & 1.263 \\
Estrato 5 & 1.220 & 1.238 & 1.260 \\
Estrato 6 & 1.215 & 1.230 & 1.083 \\
Industrial & 1.050 & 945 & 1.104 \\
Comercial & 1.037 & 1.070 & 1.095 \\
Oficial & 794 & 1.043 &
\end{tabular}

Con respecto al agua subterránea, su uso se da principalmente en las zonas de expansión urbana de la ciudad de Pereira dada la facilidad de acceso, calidad y la disminución de costos en sistemas de conducción y tratamiento. Sin embargo, en las zonas de expansión urbana donde se tiene proyectado el crecimiento de la ciudad, se encuentran ubicadas zonas estratégicas de recarga acuífera, generando de esta forma amenaza potencial tanto para calidad como para cantidad de este recurso (Paredes, Zuleta, Gómez y Lasso, 2013). En la Tabla 3 se presenta la demanda hídrica de agua subterránea en el área urbana del AMCO. 
Tabla 3. Demanda agua subterránea en el área urbana del AMCO

\begin{tabular}{l|cc}
\hline \multicolumn{1}{c|}{ Uso de agua } & $\begin{array}{c}\text { Caudal concesionado } \\
\text { l/s }\end{array}$ & $\begin{array}{c}\text { Porcentaje } \\
\%\end{array}$ \\
\hline Humano colectivo & 32,3 & 23,3 \\
Individual & 8 & 5,8 \\
Comercial & 17,7 & 12,8 \\
Industrial & 68 & 49,1 \\
Recreativo & 4,2 & 3,0 \\
Institucional & 3,02 & 2,2 \\
Estaciones de servicio & 5,3 & 3,8 \\
Total caudal concesionado & 138,52 & 100 \\
\hline
\end{tabular}

Fuente: Adaptado de Paredes, Zuleta, Gómez y Lasso (2013).

\section{C) Flujos de salida}

Con relación a los flujos de salida, los vertimientos son entregados a diferentes cuencas. El municipio de Dosquebradas vierte en promedio 7,6 MMC/año a la quebrada Dosquebradas que posteriormente desemboca al río Otún. El sistema de drenaje de la ciudad de Pereira vierte 20,7 MMC/año, distribuidos 52\% al río Consota y $48 \%$ al río Otún. El municipio de La Virginia vierte 1,3 MMC/año al río Risaralda.

Es preocupante cómo en la región se vierten las aguas residuales directamente a las fuentes de agua sin ningún tipo de tratamiento previo, generando contaminación y deterioro permanentemente. Es evidente que las corrientes locales, especialmente las quebradas Dosquebradas y Consota, se están concibiendo como receptoras de aguas residuales y no como abastecedoras del recurso para el consumo local.

En la Figura 7 se muestran los volúmenes de aguas residuales urbanas vertidas a las corrientes hídricas, destacándose que no tienen ningún tipo de tratamiento.

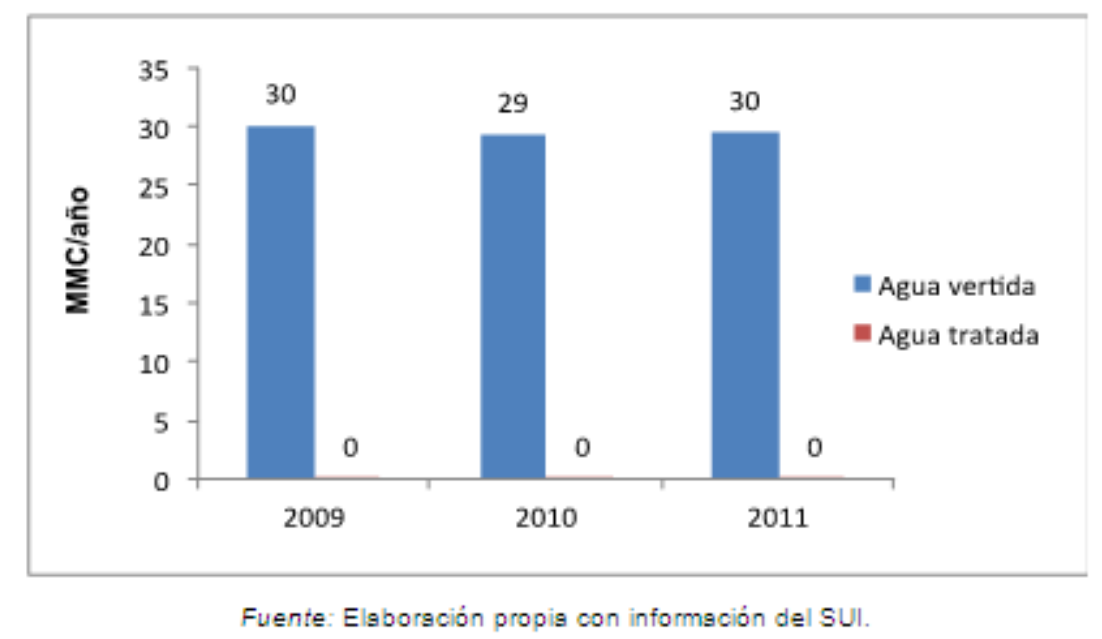

Figura 7. Volumen de agua vertida y tratada en el AMCO.

\section{Ciclo hídrico en el AMCO}

Los flujos de agua que ingresan y salen del AMCO corresponden a un proceso lineal, en el cual el agua se capta, distribuye, consume y desecha, con altas 
deficiencias, careciendo de un proceso de reintegración al ecosistema por la falta de tratamiento, conservación y eficiencia en el manejo del agua.

El modelo "Balance de agua" para el AMCO (Tabla 4) expresa tres grandes elementos: flujos de entrada, flujos intermedios y flujos de salida. Las entradas corresponden a los flujos de agua extraídos desde el medio ambiente a la ciudad; los flujos intermedios es el proceso de aprovechamiento que consiste en el uso del recurso y es el sustento de la ciudad; y las salidas representan las aguas residuales, su saneamiento, reuso y disposición final que son subproductos no requeridos de los procesos de producción y del consumo.

A partir del enunciado de la ley de la conservación de la materia, el balance de materia indica que la masa que entra en un sistema debe por lo tanto salir del sistema o acumularse dentro de él, es decir los flujos de entrada de agua han de ser cuantitativamente iguales a los flujos de salida. La ecuación 1 resuelve el balance de masas para los flujos de agua que entran y salen del AMCO.

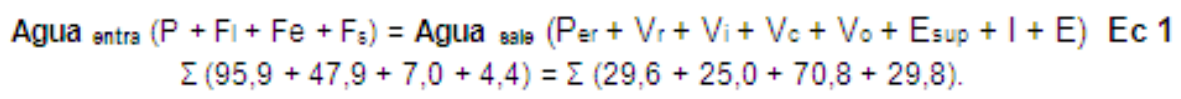

La anterior ecuación determina el flujo volumétrico de agua para el año 2011 de 155,2 MMC/año.

En la Tabla 4 se presenta detalladamente el balance de agua del AMCO para los años 2009, 2010 y 2011.

Tabla 4. Balance de agua AMCO

\begin{tabular}{|c|c|c|c|}
\hline \multicolumn{4}{|c|}{ BALANCE DE AGUA AREA METROPOLITANA CENTRO OCCIDENTE MMC/ANO } \\
\hline Variables & 2009 & 2010 & 2011 \\
\hline \multicolumn{4}{|c|}{ FLUJOS DE ENTRADA } \\
\hline $\begin{array}{l}\text { Precipitación } \\
\text { Crudglcgotado fuentes locgles suoerficigles }\end{array}$ & $\begin{array}{l}79,8 \\
50.6\end{array}$ & $\begin{array}{l}76,1 \\
46,9\end{array}$ & $\begin{array}{l}95,9 \\
47,9\end{array}$ \\
\hline Importación de sgus & 8,5 & $\begin{array}{r}4,0 \\
8,1\end{array}$ & $\begin{array}{r}4.0 \\
7,0\end{array}$ \\
\hline Extrscción sgus subterránes & 4,4 & 4,4 & 4,4 \\
\hline Totalentradas & 143,3 & 135,4 & 155,2 \\
\hline \multicolumn{4}{|c|}{ FLUJOS INTERMEDIOS } \\
\hline Demanda Residencial & 25,5 & 25,0 & 24,7 \\
\hline Demanda Industrial & 1,0 & 0,4 & 0,6 \\
\hline Demsnda Comercisl & 3,4 & 3,0 & 3,0 \\
\hline Demanda Oficisl & 1,5 & 1,4 & 1,4 \\
\hline Demenda otros sectores & 0,6 & 0,6 & 1,4 \\
\hline Totgl intermedios & 32,0 & 30,5 & 31,2 \\
\hline \multicolumn{4}{|c|}{ FLUJOS DE SALIDA } \\
\hline Pérdidss total (facy no fac) & 27,6 & 26,2 & 25,0 \\
\hline Evapotranspiración & 30,7 & 30,2 & 29,8 \\
\hline Vertimientos & 30,2 & 29,4 & 29,6 \\
\hline Escorrentis superficisl & 54,9 & 49,7 & 70,8 \\
\hline Totglsalidas & 143,3 & 135,4 & 155,2 \\
\hline
\end{tabular}

En la Figura 8 se muestra un modelo descriptivo que logra hacer una síntesis simplificada para el entendimiento de las rutas metabólicas del agua en el AMCO. 


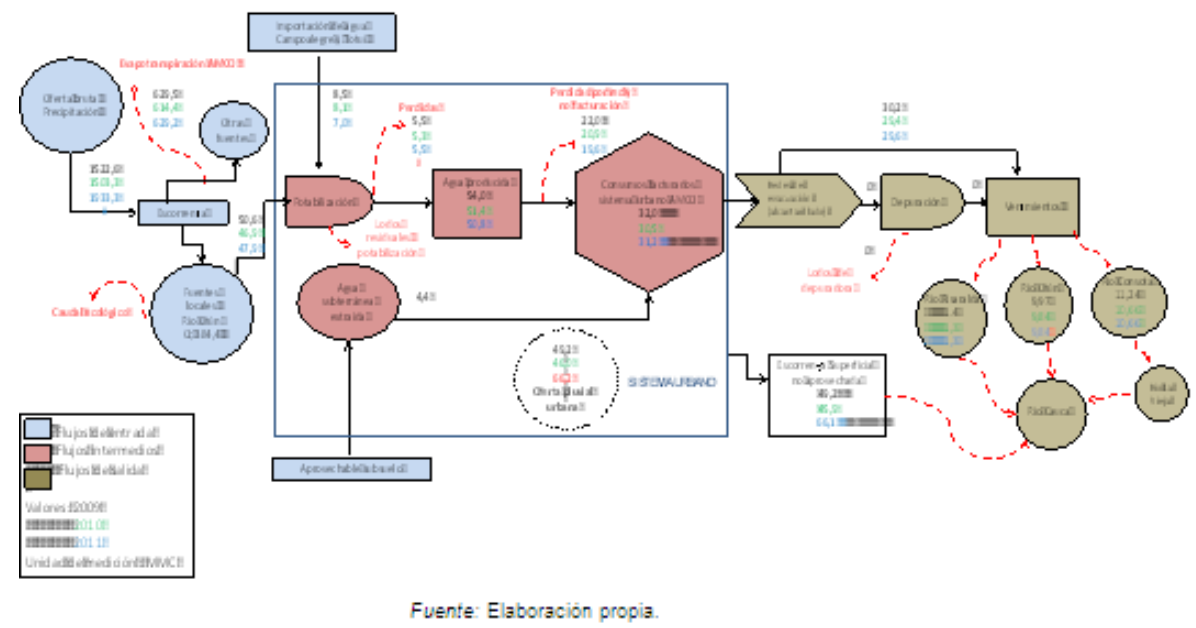

Figura 8. Modelo descriptivo metabolismo hídrico del AMCO 2009-2011.

\section{Relación oferta y demanda de agua en el AMCO}

La relación oferta-demanda de agua es una forma de medir el nivel de presión sobre los recursos hídricos. En la Tabla 5 se muestra la oferta y demanda de agua, se destaca cómo la demanda del área urbana del AMCO no supera la oferta hídrica. Por lo tanto, se afirma que en la región existe abundancia del recurso hídrico, salvo el municipio de La Virginia que puede presentar a mediano plazo dificultades para satisfacer la demanda de este recurso natural.

Tabla 5. Oferta y demanda de agua en el AMCO

\begin{tabular}{|c|c|c|c|c|}
\hline Municipio & Fuente asociada & \multicolumn{2}{|c|}{$\begin{array}{l}\text { Oferta de agua año } 2010 \\
\text { (MMC/año) }\end{array}$} & $\begin{array}{c}\text { Demanda de agua } \\
\text { año } 2010 \\
\text { (MMC/año) }\end{array}$ \\
\hline Pereira & Rio Otún & $\begin{array}{c}\text { Año medio } \\
466,30\end{array}$ & $\begin{array}{c}\text { Ano seco } \\
189,02\end{array}$ & $\begin{array}{c}\text { Estimación } \\
22,5\end{array}$ \\
\hline Dosquebradas & Rio Otún & 356,40 & 140,01 & $6,6^{\cdots}$ \\
\hline La Virginia & Rio Totuí & 15,74 & 7,21 & 1,4 \\
\hline
\end{tabular}

Morales et al. (2011) afirman que la presión por el recurso en la región es alta, como consecuencia de la tendencia de crecimiento poblacional y dado que la oferta natural no se incrementa salvo por cambios del clima regional y local, pero sí se puede disminuir por contaminación y por efectos igualmente del clima.

Es claro que uno de los retos primordiales para el desarrollo metropolitano es el manejo adecuado de los recursos hídricos. La dependencia a una única fuente local, la importación de agua, las pérdidas y el poco tratamiento a las aguas residuales urbanas pueden generar en el futuro problemas de desabastecimiento en la región.

Gestionar un modelo de agua es el desafío de los entornos urbanos: aprovechamiento de los recursos hídricos sin sobrepasar su capacidad, el ahorro significativo del agua, la reutilización del agua depurada, el aprovechamiento del 
agua lluvia y la preservación de los ecosistemas naturales. Ello es posible si el modelo se desarrolla con la intención de disminuir la presión sobre los ecosistemas que también son demandantes de agua, a través de reducir la extracción de recursos y de disminuir la carga contaminante vertida en la cuenca.

\section{CONCLUSIONES}

El análisis del metabolismo hídrico permitió conocer el estado actual del balance del agua en el AMCO. Su sostenibilidad hídrica depende tanto de su propio territorio como de un territorio tributario que le abastece de recursos y le libera de contaminantes.

Con relación a la dinámica de los servicios urbanos de agua, se carece en la región de una visión integradora del manejo del agua, pues corresponde a un proceso lineal en el cual el agua se extrae, distribuye, consume y desecha, con altas deficiencias y poco tratamiento de las aguas residuales, impidiendo su reintegración efectiva al ecosistema y a la vida urbana.

Las futuras demandas representan un gran desafío para la administración y operación del servicio, cuya gestión debe encausarse hacia nuevos paradigmas donde la conservación, la protección de las fuentes de agua y el aprovechamiento sustentable comiencen a presentar mayor relevancia.

En este estudio se encontró que la tendencia del consumo y demanda de agua es decreciente mientras que el tamaño de la población crece. Los niveles de consumo de agua muestran que en lugar de incrementarse han tenido una tendencia a la reducción. Las causas principales de este comportamiento deben estar relacionadas directamente con el incremento de la tarifa de servicio de agua. Sin embargo, se interpreta adicionalmente que dicho fenómeno podría estar además asociado con comportamientos de autorregulación de los usuarios.

\section{RECOMENDACIONES}

Debido al potencial no aprovechado de aguas pluviales, es necesario identificar los posibles usos de las aguas de lluvia para su eventual aprovechamiento como fuente alterna de abastecimiento, reduciendo la dependencia a las fuentes locales y externas.

Es necesario contar en la región metropolitana con un organismo que informe de manera precisa el estado ambiental urbano del recurso hídrico en el territorio y analice las dinámicas y procesos que se dan en la ciudad con el objetivo de mejorar su eficiencia y disminuir su impacto.

El área metropolitana necesita un sistema de información ambiental con información continua en el tiempo, de alta calidad y analizada; al alcance de quienes la requieran: la comunidad, los gestores, los tomadores de decisiones e instituciones. 


\section{AGRADECIMIENTOS}

Los autores queremos expresar nuestros agradecimientos a la Universidad Tecnológica de Pereira y al Departamento Administrativo de Ciencia, Tecnología e Innovación -Colciencias-, por el apoyo técnico y financiero del proyecto "Demanda de bienes y servicios ecosistémicos como determinantes de la sustentabilidad en áreas metropolitanas. Estudio de caso: Pereira, Colombia", del cual se deriva esta publicación.

\section{REFERENCIAS}

- Aguas y Aguas de Pereira. (2009). Informe de gestión. Recuperado el 18 de noviembre de 2012 de http://www.aguasyaguas.com.co/sitio/index.php?option=com_phoca download\&view=category\&id=2\&presets $=$ preset5\&ltemid $=183$

- Arbués García, F., Barberán Orti, R. y Villanúa Martin, I. (2008). Tamaño de los hogares y demanda de agua residencial una aproximación empírica. III Congreso de Aerna. Palma de Mayorca.

- Banco Mundial. (2012). Gestión integral de aguas urbanas. Recuperado el 16 de marzo de 2013 de www.worldbank.org

- Brunner, P. (2007). Reshaping urban metabolism. Industrial Ecology, 11(2), 11-13.

- Calderón, R. (2011). Modelo de metabolismo energético, a partir del consumo eléctrico de la vivienda económica de Mexicali, B.C. Tesis de Doctorado en Planeación y Desarrollo Sustentable. Mexicali, Baja California: Universidad Autónoma de Baja California, Facultad de Arquitectura y Diseño. Instituto de Investigaciones Sociales.

- Campbell, H.E., Larson, E.H., Johnson, R.M. y Waits, M.J. (1999). Some best bets in residential water conservation. Results of multivariate regressiona analysis, City of Phoenix, 1990-1996. Recuperado el 24 de julio de 2013 de http://morrisoninstitute.asu.edul publications-reports/BestBetsInH2OConserv-ResultsMultivarRegrPhx90-96

- Cárdenas, N. (2013). Análisis a la gestión del plan de saneamiento hídrico de Pereira desde la perspectiva integral del recurso, haciendo uso de weap como sistema soporte de decisión. Tesis de pregrado. Universidad Tecnologica de Pereira, Pereira.

- Delgado Ramos, G.C., Campos Chávez, C. y Rentería Juárez, P. (2012). Cambio climático y el metabolismo urbano de las megaurbes latinoamericanas. Hábitat Sustentable, 2(1), 2-25.

- Departamento Administrativo Nacional de Estadística. (24 de noviembre de 2005). Proyecciones de población municipales por área. Recuperado en agosto de 2012 de www.dane.gov.co

- Díaz Álvarez, C.J. (2011). Metabolismo de la ciudad de Bogotá D.C: una herramienta para el análisis de la sostenibilidad ambiental urbana. Maestría tesis. Universidad Nacional de Colombia.

- Gandy, M. (2004). Rethinking urban metabolism: water, space and the modern city. City, 8(3), 363-379.

- Girardet, H. (2004). Cities, People Planet. First Edition. John Wiley \& Sons.

- Gómez, B. (1980). Balance de agua en redes de distribución. Recuperado el 21 de agosto de 2013 de http://www.bvsde.paho.org/bvsacd/scan/011000/011000-06.pdf 
- Hermanowicz, W. y Asano, T. (1999). Abel Wolman's "the metabolism of cities" revisited: A case for water recycling and reuse. Water Science and Technology, 40(4-5), 29-26.

- Hoeskstra, A.Y. y Chapagain, A.K.. (2008). Globalization of water. Sharing the planet's freshwater resources. Blackwell Publishing.

- March, H. y Sauri, D. (2010). Flujos de agua, flujos de capital: sistemas de abastecimientos y gobernanza del agua en Madrid y Barcelona. Investigaciones Geográficas, 51, 7-26.

- Morales, T., Flórez, M.T. y Ramírez, C.L. (2011). Indicadores ambientales para el estudio de la dinámica del recurso hídrico en Risaralda. Pereira: Universidad Tecnológica de Pereira.

- Navarro Chaparro, S. (2010). La problemática del agua urbana en la ciudad de Tijuana, Baja California y algunas alternativas para una gestión sustentable. Tesis de Maestría en Administración Integral del Ambiente. México, Departamento de Urbanismo, El Colegio de la Frontera Norte, A.C. y Centro de Investigación Científica y Educación Superior de Ensenada.

- Niza, S., Rosado, L., \& Ferrao, P. (2009). Urban Metabolism. Methodological Advances in Urban Material Flow Accounting Based on the Lisbon Case Study. Industrial Ecology , 13 (3), 384-405.

- ONU-HÁBITAT. (2009). Informe mundial sobre asentamientos humanos, 2009. Recuperado el 6 de junio de 2013 de http://www.unhabitat.org/documents/grhs09/k0952834s.pdf

(2012). Estado de las ciudades de América Latina y el Caribe 2012. Rumbo a una nueva transición urbana. Brasil.

- Paredes, D., Zuleta, D., Gómez, M.A. y Lasso, J. (2013). Impactos del crecimiento urbanístico y alternativas de manejo de las aguas subterráneas en la zona de expansión occidental del municipio de Pereira. (G. G. Hídrico, Ed.). Pereira: Universidad Tecnológica de Pereira.

- PNuma. (2000). América Latina y el Caribe. Perspectiva del medio ambiente. Costa Rica.

- Quintana, A.P. (2009). Rasgos del conflicto por la gestión colectiva del agua en Dosquebradas - Risaralda, Colombia. Recuperado de http://www.cap-net-esp.org/document/document/146/

124_Rasgos_del_conflicto_gestion_del_agua_Colombia.doc

- Serviciudad ESP. (2009). Informe de gestión. Recuperado el 22 de noviembre de 2012 de http://www.serviciudad.gov.co/web/index.php?option=com_content\& view= article $\& i d=60 \&$ Itemid $=73$

- Sistema Único de Información de Servicios Públicos (SUI). (2009). Consulta de información, acueducto. Recuperado el 10 de abril de 2013 de http://www.sui.gov.co/suibase/operaciones/facturacionAAA.htm

- Stimson, R., Westem, J., Mullins, P. y Simpson, R. (1999). Urban metabolism as a framework for investigating quality of life and sustainable development in the Brisbane - Southeast Queensland Metro Region. En: Yuan, L.L., Yuen, B. y Low, C. (eds.). Quality of life: critical issues and options (pp. 143-168). Singapur: Sch. de la Construcción y Bienes Raíces, National University de Singapur.

- Thériault, J. y Laroche, A.-M. (2009). Evaluation of the urban hydrologic metabolism of the Greater Moncton region, New Brunswick. Canadian Water Resources, 34(3), 255-268. 
1. Administradora Ambiental. Candidata a Magíster en Gestión Ambiental Metropolitana. Co-investigadora Grupo de Investigación Gestión Ambiental Territorial, Facultad de Ciencias Ambientales, Universidad Tecnológica de Pereira (UTP), Colombia. La Julita, Pereira, Risaralda, Colombia. A.A.: 097. maria.isabel681@gmail.com

2. Administrador Ambiental. Magíster en Investigaciones de Operaciones y Estadísticas. Doctor en Ciencia y Tecnología Ambientales. Investigador Grupo de Investigación Gestión Ambiental Territorial, Facultad de Ciencias Ambientales, Universidad Tecnológica de Pereira (UTP), Colombia. La Julita, Pereira, Risaralda, Colombia. A.A.: 097.

Investigador Grupo de Investigación Sostenipra (UAB-IRTA-Inèdit). Institute of Environmental Science and Technology (ICTA). Universitat Autònoma de Barcelona (UAB). School of Engineering (EE), Campus de la UAB, Bellaterra (Cerdanyola del Vallès) 08193 Barcelona, Catalonia, Spain. tito@utp.edu.co

3. Ingeniero Sanitario. Magíster en Ingeniería Sanitaria y Ambiental. Doctor en Ingeniería. Investigador Grupo de Investigación en Agua y Saneamiento. Decano Facultad de Ciencias Ambientales, Universidad Tecnológica de Pereira (UTP), Colombia. La Julita, Pereira, Risaralda, Colombia. A.A.: 097. jhguerre@utp.edu.co

Para citar este artículo: García-Serna, M.I., Morales-Pinzón, T., Guerrero, Erazo, J. (2014). Análisis de flujos de agua en áreas metropolitanas desde la perspectiva del metabolismo urbano. Revista Luna Azul, 39, 234-249. Recuperado de http://lunazul.ucaldas.edu.co/index.php?option=content\&task=view\&id=957 